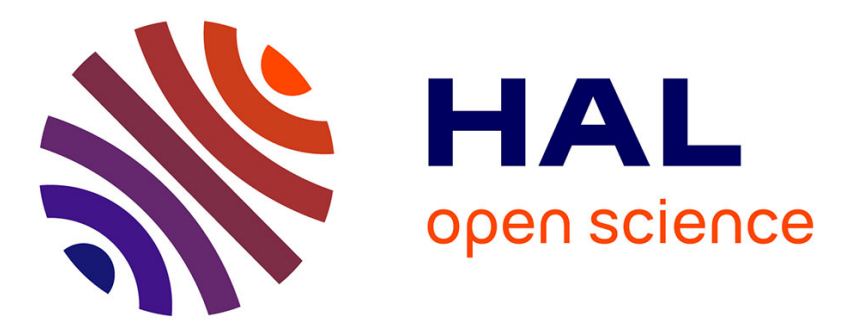

\title{
Use of Integrated Modeling to Enhance Estimates of Population Dynamics Obtained from Limited Data
}

Michael Schaub, Olivier Gimenez, Antoine Sierro, Raphaël Arlettaz

\section{To cite this version:}

Michael Schaub, Olivier Gimenez, Antoine Sierro, Raphaël Arlettaz. Use of Integrated Modeling to Enhance Estimates of Population Dynamics Obtained from Limited Data. Conservation Biology, 2007, 21 (4), pp.945-955. 10.1111/j.1523-1739.2007.00743.x * hal-03515125

\section{HAL Id: hal-03515125 \\ https://hal.science/hal-03515125}

Submitted on 6 Jan 2022

HAL is a multi-disciplinary open access archive for the deposit and dissemination of scientific research documents, whether they are published or not. The documents may come from teaching and research institutions in France or abroad, or from public or private research centers.
L'archive ouverte pluridisciplinaire $\mathbf{H A L}$, est destinée au dépôt et à la diffusion de documents scientifiques de niveau recherche, publiés ou non, émanant des établissements d'enseignement et de recherche français ou étrangers, des laboratoires publics ou privés. 


\section{Use of Integrated Modeling to Enhance Estimates of Population}

\section{Dynamics Obtained from Limited Data}

Michael Schaub ${ }^{1,2}$, Olivier Gimenez ${ }^{3}$, Antoine Sierro ${ }^{2,4}, \&$ Raphaël Arlettaz ${ }^{1,2,4}$

1. Zoological Institute - Conservation Biology, University of Bern, Baltzerstrasse 6, CH3012 Bern, Switzerland

2. Swiss Ornithological Institute, CH-6204 Sempach, Switzerland

3. Centre for Research into Ecological and Environmental Modelling, University of St Andrews, St Andrews, The Observatory, Buchanan Gardens, KY16 9LZ, Scotland

4. Valais Bat Conservation Group, Nature Centre, $\mathrm{CH}-3970$ Salgesch, Switzerland Running title: Integrated population modeling of bats

7342 words (from abstract through to literature, inclusive) You need to cut at least 300 words to come in at just over 7000

Correspondence author: Michael Schaub

Zoological Institute - Conservation Biology University of Bern

Baltzerstrasse 6

CH-3012 Bern

Switzerland

Fax: +41316313163 E-mail: michael.schaub@nat.unibe.ch 


\begin{abstract}
Demographic data of rare and endangered species are often too sparse to estimate vital rates with sufficient precision for understanding population growth and decline. Yet, the combination of different sources of demographic data into one statistical model holds promise. We applied Bayesian integrated population modeling to demographic data from a colony of the endangered greater horseshoe bats (Rhinolophus ferrumequinum). Available data were the number of subadults and adults emerging from the colony roost at dusk, the number newborns from 1991 to 2005, as well as recapture data of subadults and adults from 2004 and 2005. Survival rates did not differ between sexes and demographic rates remained constant across time. The greater horseshoe bat is a long-lived species with high survival rates (first year: 0.49 [SD: 0.06], adults: 0.91 [SD: 0.02]) and low fecundity (0.74 [SD: 0.12]). The yearly average population growth was 4.4\%. Had we analyzed each data set separately, we would not have been able to estimate fecundity, the estimates of survival would have been less precise and the estimate of population growth biased. Our results demonstrate that integrated models are suitable for obtaining crucial demographic information from limited data.
\end{abstract}

Key words: Bats, demography, monitoring, Rhinolophus ferrumequinum, species conservation, state-space model, integrated population modeling. 


\section{Introduction}

In the face of the rapid global biodiversity loss, the declining population paradigm has become central in conservation biology (Caughley 1994). It aims to identify the reasons for the decline of a specific population. There are several possible approaches to identify causes of population declines, the most popular being demographic analysis (Norris 2004). Central to demographic analysis is the estimation of demographic parameters (survival, reproduction, immigration, emigration) and the exploration of the relationship between variation of these parameters and population growth rate. This approach requires that the strength of impact of a particular demographic parameter on the population growth rate be assessed with a sensitivity analysis (van Groenendael et al. 1988). The final step is to identify environmental- and density-related factors that affect those demographic parameters that are most relevant to variation of population growth rate.

Although a demographic analysis is a powerful tool, it is difficult to apply for many species because the necessary, detailed demographic data are often not available and because inferences based on demographic analyses with insufficient data can be flawed (Doak et al. 2005). Even worse, data are frequently insufficient for declining or rare species (Beissinger 2002). Data of endangered species can be scarce because species' sample size is naturally low and researchers hesitate to catch and mark individuals, a prerequisite to obtain most demographic data. Typically, population size is usually well documented, whereas few longitudinal data on individuals (demographic data) are available. In such a situation, it is crucial to make most efficient use of all available data.

Recently developed integrated population models (Besbeas et al. 2002; Buckland et al. 2004; Brooks et al. 2004; Besbeas et al. 2005) are likely to prove useful for detailed demographic analyses. Once again, Buckland et al. 2004 do not deal with integrated population modeling. . This ref. should be deleted. These models combine population counts and 
demographic data in a single model, which thus allows the estimation of demographic parameters and the prediction of population trajectories. Because the population counts also contain information about all demographic parameters of the population under study, the estimates of the demographic parameters become considerably more precise and otherwise inestimable demographic parameters can become estimable (Besbeas et al. 2002). Thus, one might hope that such models could be used to estimate vital parameters even if few demographic data were available. So far, these models have been applied mainly by statisticians to species without deficiency of demographic data. We applied this modeling approach to a small data set on a rare species and evaluated its usefulness for demographic analysis.

We will use data from a bat as a case study. Bats are declining globally (Mickleburgh et al. 2002), but few detailed demographic analyses have been performed so far. Even single demographic parameters have been poorly studied, as exemplified by the few estimates of survival rates obtained with reliable statistical methods (Boyd \& Stebbings 1989; Gerell \& Lundberg 1990; Hoyle et al. 2001; Sendor \& Simon 2003; O'Shea et al. 2004; Pryde et al. 2005). Population dynamics of bats is difficult to study for three reasons. First, the most popular methods to assess whether a bat population is changing is counting individuals at nursery colonies (Warren \& Witter 2002). Such colonies mostly consist of reproducing females, with some nonreproducing subadult females and males (Ransome 1990; Neuweiler 2000). Because sexing and ageing of bats is impossible unless they are captured, the fraction of reproducing females remains unknown. If this fraction changes over time, annual counts at nursery colonies will not reflect population changes accurately. Second, estimation of fecundity (number of offspring a mature female is producing in a year) is difficult. In many bat species females give birth to a single offspring per year, but little is known about regularity of breeding from year to year in individual females (Racey 1982). Because females that skip a breeding event are usually not at the nursery colonies (R. Arlettaz, unpublished data on mouse-eared bats), the ratio of the 
number of newborn to the number of mature females present at the colony appears to be an unreliable estimate of fecundity. Third, catching and marking bats is not considered ethical anymore because catching may disturb them and rings may affect foraging performance (Norman et al. 1999) or cause injury (Baker et al. 2001). Therefore, good individual longitudinal data are not available for many bat species, which precludes the estimation of survival probabilities. Because basic demographic information is lacking for many bat species, it is difficult to devise conservation actions for them.

We sought to demonstrate the flexibility and power of integrated population models to estimate demographic parameters from sparse data, with a relictual colony of greater horseshoe bats (Rhinolophus ferrumequinum). We tested whether demographic parameters changed over time and estimated the rate of change of the study population. This allowed us to identify roughly whether a gradual change of a demographic parameter resulted in a population change and to evaluate the current state of the population. Finally, we compared the rate of population change obtained from the counts alone with the estimate from the population model and considered the general benefits of an integrated population model and how it could be used in demographic monitoring.

\section{Data and methods}

\section{Study species}

The greater horseshoe bat is one of the most threatened bat species of Central and Western Europe (Stebbings 1988). In Switzerland it is listed as endangered (Duelli 1994), and there are only two known nursery colonies, one in eastern Switzerland (Grisons) and one in southwestern Switzerland (Valais). Results of studies of populations at the northern margin of the species distribution range in Great Britain show that females start reproducing at 2 or 3 years, that survival rate during the first year of life is $53 \%$, that survival of adult females is in the range of 
$67-91 \%$, and that the breeding probability (probability of an adult female reproducing in a given year) is about 0.9 (Ransome 1990, 1995). Moreover, population dynamics are driven mainly by the variation of offspring production (Ransome 1989), which itself is related to weather conditions in spring (Ransome \& McOwat 1994). Yet, the results of these studies rely on the untested assumption that the fate of each bat is known with certainty.

\section{Data collection}

We studied a greater horseshoe bat colony in the attics of a twelfth-century chapel in Vex, Valais, Switzerland $\left(46^{\circ} 13^{\prime} \mathrm{N}, 7^{\circ} 24^{\prime} \mathrm{E}\right)$. This colony was isolated from the other known Swiss population and from the Italian and French populations by high mountain ridges (Arlettaz et al. 1997). This population was discovered in 1986 , just prior to the renovation of the building (1987-1988), which was planned in compliance with the requirements for the colony's preservation (Arlettaz et al. 1997).

With the exception of 2 years, we counted from 1987 onwards in every year the number of individuals emerging from the roost at dusk, prior to the onset of parturition. Since 1991, during the first weeks after parturition, we visited the attics after the emergence of subadult and adult bats - when young were left unattended - and counted and ringed most newborns (260 out of 285 newborns ringed in total). This way, adults were never disturbed at the colony roost. In 2004 and 2005, shortly before parturition, which corresponded to the period when the census was carried out and when the population peaked (R. Arlettaz and A. Sierro, unpublished data), we blocked the main entrance and captured, in 1 day at daylight, the entire population. Ring number and sex of the bats $\left(n_{2004}=54, n_{2005}=52\right)$ were recorded. This massive capture did not affect the number of bats that returned to the roost on the following days nor reproduction. Indeed, 2004 and 2005 yielded an unequalled number of offspring. Because we only began 
ringing newborn bats in 1991 only, we considered for our analysis the period from 1991 to 2005, although in the year 1991 no population count was carried out.

\section{Integrated population model}

We used an integrated population model, which integrated different kinds of data into a common model, to study the population dynamics of the greater horseshoe bat (Besbeas et al. 2002). We describe the development of the population size with two linked processes, namely the state and the observation process. The state process describes the true, but unknown, size of the population at different times, and the observation process links the size of the population to the observed part of the population during the surveys. The state process is described by different demographic parameters (survival, fecundity), which were estimated each with separate probabilistic models. To allow for maximal flexibility regarding the model assumptions, we used Markov chain Monte Carlo (MCMC) methods within a Bayesian framework to fit the model (Brooks et al. 2004; Maunder 2004).

Because most greater horseshoe bats do not start to reproduce in their first year of life (Ransome 1990; R.A. unpublished data: only 2 out of 14 females were pregnant in their first year of life), we considered for each sex two age classes in our model: individuals aged 1 year (subadults) and individuals older than 1 year (adults). To describe the model, we start with the likelihoods for the different demographic parameters and the population sizes and then we show how they were linked and how they were estimated in the integrated population model.

\section{Likelihood for estimation of local survival rates}

To estimate local survival probabilities $\left(\phi_{x, t}^{k}\right.$, probability to survive and not to emigrate permanently from the population between year $t$ and year $t+1$ of individuals of sex $k$ and age class $x$ ) from individual capture-recapture data, we used the Cormack-Jolly-Seber (CJS) model 
(Lebreton et al. 1992). The frequency of individual encounter histories followed a multinomial distribution with cells that were functions of the two parameters local survival $\left(\phi_{x, t}^{k}\right)$ and recapture $\left(p_{x, t}^{k}\right)$ probabilities (notation as above). The formulation of the likelihood of this model was straightforward and is described in many papers (e.g., Lebreton et al. 1992). Because we only had recaptures from the last 2 years, we set the recapture probabilities for all but the last 2 years equal to zero.

\section{Likelihood for estimation of fecundity}

We derived fecundity from the yearly counts of the newborn. The fecundity rate $\left(f_{t}\right)$ was defined as the number of offspring produced per mature female in year $t$. We assumed that the number of newborns at time $t\left(J_{t}\right)$ followed a Poisson distribution and depended on the number of adult females $\left(N_{a, t}^{f}\right)$ and the fecundity rate $\left(f_{t}\right)$, thus $J_{t} \sim \operatorname{Po}\left(N_{a, t}^{f} f_{t}\right)$.

\section{Likelihood of the estimation of population sizes}

Our population count of horseshoe bats emerging from the nursery colony at dusk consisted of subadults and adults from both sexes. We knew that all reproducing females and a varying fraction of males and subadult, nonreproducing females are present at nursery colonies (Gaisler 1966). However, flying bats cannot be aged or sexed. To include these counts in a state-space model, we needed to know the proportion of the four sex and age classes. We estimated these parameters as follows. First, we noted that the recapture probability estimated with the CJS model was composed of two parts, the probability that a bat would be recaptured given presence in the colony and the probability it would be present in the colony. This partition is strictly true if the probability of being present is random (Burnham 1993; Schaub et al. 2004). Because we captured all bats that were in the colony at time of capture, the true recapture probability was 1. 
Assuming that the probability of being present at the colony was random, the estimated recapture rate (from the CJS model) was equal to the probability to be in the colony. Because we only had such estimates for each sex and age class from the last 2 years, we calculated the geometric mean of the 2 values and considered these mean values the same over the duration of the study. Specifically, the probability that an individual of age $x$ and sex $k$ was present in the colony was calculated as $\tau_{x}^{k}=\sqrt{p_{x, 2004}^{k} p_{x, 2005}^{k}}$. The population count in year $t\left(y_{t}\right)$ was then related to the actual population sizes with a normal distribution as

$$
y_{t} \sim N\left(\tau_{1}^{f} N_{1, t}^{f}+\tau_{a}^{f} N_{a, t}^{f}+\tau_{1}^{m} N_{1, t}^{m}+\tau_{a}^{m} N_{a, t}^{m}, \sigma_{y}^{2}\right),
$$

where $N_{x, t}^{k}$ is the number of individuals of sex $k$ in age class $x$ in year $t$ and the variance $\sigma_{y}^{2}$ quantifies the counting error. Superscripts $m$ and $f$ denote male and female, respectively, subscript 1 denotes the subadult age class and subscript $a$ the adult age class.

\section{Linking demography and population size}

The next step was to link the demographic parameters with the number of individuals in each sex and age class. We used a Leslie matrix that described the transition probabilities between sex and age classes from one year to the next and that was parameterized with the survival rates and fecundity. We assumed that annual survival rate during the first year of life was different from annual survival later on and that the latter did not change further with age. Furthermore, we assumed that all females began to reproduce when they were 2 years old and that the sex ratio of the newborn was even. The latter assumption was supported by the sex ratio of the newborn with known sex $(52.5 \%$ females, $n=260)$. To account for demographic stochasticity, we described the change of each population segment with Poisson and binomial distributions. Specifically, we modeled the number of subadults in year $t+1$ according to a Poisson distribution:

$$
N_{1, t+1}^{f} \sim \operatorname{Po}\left(N_{a, t}^{f} \frac{1}{2} f_{t} \phi_{1, t}^{f}\right) \text { for females and }
$$




$$
N_{1, t+1}^{m} \sim \operatorname{Po}\left(N_{a, t}^{f} \frac{1}{2} f_{t} \phi_{1, t}^{m}\right) \text { for males. }
$$

The number of adults in year $t+1$ was distributed as binomial:

$$
\begin{aligned}
& N_{a, t+1}^{f} \sim \operatorname{Bin}\left(N_{a, t}^{f}+N_{1, t}^{f}, \phi_{a, t}^{f}\right) \text { for females and } \\
& N_{a, t+1}^{m} \sim \operatorname{Bin}\left(N_{a, t}^{m}+N_{1, t}^{m}, \phi_{a, t}^{m}\right) \text { for males. }
\end{aligned}
$$

\section{The integrated model}

The joint likelihood of the integrated model was the product of the likelihoods of each part. The different likelihoods (survival, fecundity, and population size) had parameters in common, as illustrated graphically by the directed acyclic graph (Fig. 1).

The frequentist approach to estimate the unknown parameters would require maximizing the joint likelihood, which involves a high dimensional integral corresponding to the state-space model (Buckland et al. 2004) that could be handled by using Kalman filtering (Besbeas et al. 2002, 2003). This approach requires strong assumptions such as linearity and normality in the state process equations (Besbeas et al. 2002, 2003). The Bayesian approach combines the joint likelihood with prior probability distributions of the parameters to obtain the posterior distribution of the parameters of interest based on Bayes' theorem. We used Markov chain Monte Carlo (MCMC) methods to simulate observations from the posterior distributions. This allowed us to cope with complex integrals that are involved in this likelihood when the linear and/or normal assumptions have to be relaxed (Brooks et al. 2004). This part reads much better than before! The MCMC sampling scheme simulates values for the unknown quantities of interest following a Markov chain in which the stationary distribution is the needed posterior distribution (e.g., Brooks 1998). A burn-in period ensures that the Markov chain has reached its stationary distribution. Inference is then based on the remaining simulated values by computing numerical summaries such as empirical means and confidence intervals for the quantities of interest. 


\section{Candidate models, prior distributions and derived parameters}

We were interested in whether survival and/or fecundity rates changed gradually over time. Thus, we considered linear relationships

$$
\begin{aligned}
& \operatorname{logit}\left(\phi_{x, t}^{k}\right)=\alpha_{x}^{k}+\beta_{x}^{k} \text { year and } \\
& \log \left(f_{t}^{k}\right)=\gamma^{k}+\delta^{k} \text { year, }
\end{aligned}
$$

where year is a continuous variable. Furthermore, we included models in which survival was not sex specific, and we applied models where these demographic rates were constant $\left(\beta_{x}^{k}\right.$ and/or $\delta^{k}$ set equal to 0 ). We used different combinations that resulted in a set of 15 candidate models. We also included a more general model with time-dependent parameters. We used the deviance information criterion (DIC; Spiegelhalter et al. 2002) to rank these models according to their support by the data. The DIC quantifies the trade-off between quality of fit and model complexity and takes prior information into account. It is calculated as,

$$
D I C=-2 \log L(\text { data } \mid \theta)-2 p_{D}
$$

where $\log L($ data $\mid \theta)$ is the deviance and $p_{D}$ is the effective number of parameters. In the case of uninformative priors the DIC is approximately equal to the well known Akaike information criterion (Burnham \& Anderson 1998). The model with the smallest DIC is selected as the best.

For the complete specification of the Bayesian model, prior distributions for all parameters need to be chosen. Because a priori knowledge about demographic parameters in the greater horseshoe bat is limited, we choose uninformative priors for all parameters we intended to estimate. Specifically, we used vague normal priors $(\mathrm{N}(0,1000))$ for the regression parameters $(\alpha, \beta, \gamma, \delta)$, vague beta priors $(\beta(1,1)$ equivalent to a uniform distribution between 0 and 1$)$ for the recapture rates, and vague normal priors truncated to positive values for the initial statespecific population sizes $\left(\mathrm{N}(10,10000)\right.$ for $N_{1,1}^{k}$, and $\mathrm{N}(20,10000)$ for $\left.N_{a, 1}^{k}\right)$. 
To calculate the posterior distributions of the parameters of interest, we used MCMC simulations implemented in program WinBUGS (Spiegelhalter et al. 2004) that we executed from R (R Development Core Team 2004) with package R2WinBUGS (Sturtz et al. 2005). (See Supplementary Material for information on the code for fitting the model.) Initial trials showed that convergence occurred quickly (after about 5000 iterations) as evidenced by the Brooks Rubin - Gelman diagnostic (Brooks \& Gelman 1998). Therefore, for the main analysis we ran the MCMC algorithm for 1,100,000 iterations, discarded the first 100,000 iterations as burn in, and thinned the remainder to 1 in every 10 iteration.

We calculated some derived parameters to characterize the population. The annual growth rate $\lambda_{t}$ was calculated as the ratio of the number of females in year $t$ to the number of females in year $t+1$. The averaged population growth rate over the study period was calculated as the geometric mean of all year-specific values. The size of the complete population prior to birth was the sum of the members from all sex and age classes in that year. We calculated the probability that subadult males and females would be differentially present at the nursery colony as the quotient of the number of times the difference between these quantities was larger than zero in the MCMC samples and of the total number of MCMC samples. These quantities were easily obtained as by-products of the MCMC iterations.

\section{Results}

The number of individuals we counted each year increased over time from 27 in the year 1992 to 59 in the year 2005, and the number of newborns increased from 11 in year 1991 to 33 in year 2005 (Fig. 2).

Model selection revealed that the simplest model with constant survival and fecundity rates and no sex dependence had the strongest support (Table 1). However, the difference in DIC to the next-best candidate models was small, and thus there remained considerable uncertainty 
about the structure of the best model. The models that were closest to the most parsimonious contained sex effects on survival and a linear trend in fecundity.

The estimated demographic rates showed that the greater horseshoe bat is a long-lived species with average adult survival of 0.91 (Table 2). The fecundity rate was rather low with about $74 \%$ of all adult females that reproduce in a year. As expected, the probability for an individual would be present at the roost was lowest in adult males (0.28) and highest in adult females (0.92). The probability that subadult males would be present at the colony tended to be lower (0.68) than that of subadult females (0.82), but this difference was not significant (difference: 0.14 , SD: 0.17 , probability that the difference was different from zero: 0.20 ; Table 2).

All estimated demographic parameters for males had larger standard deviations than the corresponding parameters for females (Table 2). This was also apparent for the sex-specific juvenile survival rates from the second-best model: the standard deviation for the males $(0.142)$ was twice as large as the standard deviation of the females (0.071), although the means were almost identical (males, 0.506; females, 0.496).

The estimated population sizes of females and subadult males were increasing over time (Fig. 3). The estimates for 1991, in particular those of the subadults were not reliable, and the estimates of the adult males were generally imprecise. The size of the complete population (sum of both age classes and sexes) increased from 70 individuals (SD 21) in the year 1992 to 96 individuals (SD 12) in the year 2005. Because the estimation of the males was less reliable than that of the females, we considered that the total population size was twice the total number of females. This can reasonably be assumed given the even sex ratio at birth and no sex-specific differences in local survival probabilities. Under this assumption, the model estimated that the entire population had increased from 51 (SD 9) to 92 (SD 10) individuals between 1992 and 2005. 
The mean annual population growth rate calculated as the geometric mean of the yearspecific population growth rates of the adult females indicated positive population growth (1.044, SD: 0.014, 95\% credible interval: 1.018 - 1.073; range: 0.979 [SD: 0.077] - 1.085 [SD: 0.073]). The mean population growth rate obtained by the counts was higher (1.062), and the variation between years was larger (Fig. 4).

To assess the impact of model selection uncertainty on our conclusions, we considered the estimates of each demographic parameter across all models with $\Delta \mathrm{DIC}<2$ and calculated the difference between the maximum and the minimum value. These differences were small for all parameters (survival rates: $<0.04$; fecundity: $<0.06$, population sizes: $<3.5$; population growth rate: 0.018 ) indicating that our conclusions were robust despite considerable model selection uncertainty. OK, neat idea ;-)

\section{Discussion}

The combination of different sources of information in a Bayesian framework through the product of three different likelihoods applied to a sparse data set of an endangered bat species was helpful for estimating demographic rates and population size. In addition we could test hypotheses regarding the variation of demographic rates. We found that the greater horseshoe bat colony at Vex has increased by $4.4 \%$ each year, that the species is long-lived with survival rates of about $50 \%$ in the first year and of about $90 \%$ when adult, and that females reproduced successfully in about 3 out of 4 years.

Our estimated survival rates for juvenile greater horseshoe bat $(0.49)$ were similar to those of Ransome (1990). In contrast, our estimates for the adults (0.91) were higher on average than Ransome's (1990) (0.66 - 0.91). This discrepancy is likely a consequence of calculating return instead of local survival rate; the former is unreliable when trappability is not $100 \%$ (Martin et al. 1995). Reliable estimates of survival rates from other bat species are scarce. In all 
species studied so far adult survival is lower than 0.9 (Boyd \& Stebbings 1989; Gerell \& Lundberg 1990; Hoyle et al. 2001; Sendor \& Simon 2003; O'Shea et al. 2004; Pryde et al. 2005). Among bats, different life histories are likely to exist with a cline from short-lived species that are relatively more productive to relatively longer-lived species that are less productive, as found in other groups of vertebrates (Saether \& Bakke 2000). Although basic demographic information of many other bat species must be known to make strong conclusions, there is evidence that the greater horseshoe bat is at the long-lived end of this continuum (Gaisler 1989).

Comparison of fecundity with other bat species is difficult because such estimates are largely lacking. As regards greater horseshoe bats, Ransome's (1995) fecundity estimate of 0.9 is likely an overestimation given that the ratio of females that showed signs of reproduction to all adult females captured in a given year was used. If nonreproducing females do not use the nursery roost where captures are carried out, for instance because they skip reproduction during certain years (this study), then this estimate of fecundity is biased high. The combination of a high survival rate (0.9) and high fecundity (Ransome's 0.9) would have lead to an unrealistic doubling of the British population of greater horseshoe bats within $\sim 5$ years assuming that all individuals started to reproduce only at 3 years of age. In contrast, our model actually accounted for temporary absence from the colony.

We can only speculate at this stage about why the population in Vex has increased. Because there is no strong evidence that the demographic parameters have changed over time, it is likely that good conditions already prevailed because of the bat-friendly restoration of the building in 1989 when we started to monitor the colony. This conservation measure may have been crucial for the colony's increase. However, a model in which fecundity gradually increased was not very far from the best model (Table 1). Thus, there remains uncertainty about whether the population increase was supported by (it might not be clear what you mean by supported by) increasing fecundity. With sparse data, subtle patterns are difficult to recognize. 
If the population increases further, we can expect that density-dependent mechanisms may start to operate. These may affect all demographic parameters, yet we expect that they will become evident first in emigration. This is because, historically, the greater horseshoe bat was widespread in Valais, and there is some potential for recolonization of abandoned colonies if local foraging habitat is still suitable. If emigration were to become stronger, this would be evident in a decrease of juvenile local survival.

The population sizes for 1991 were not estimated reliably, probably because there was no census carried out in this year. More generally, the fact that obviously no information is available prior to the beginning of the study may explain why the estimate from the first year is less reliable than those of later years. Technically, it is indeed very difficult to provide initial values that are required to start the fitting algorithm close enough to the true population sizes. I MUST CONFESS THAT THIS POINT IS NOT CLEAR TO ME EITHER. I KNOW THAT PANAGIOTIS (BESBEAS) IS WORKING ON DIFFERENT TECHNIQUES TO PROVIDE GOOD GUESS FOR THOSE INITIAL VALUES IN ORDER TO BURN AS LESS AS POSSIBLE THE INFORMATION IN THE BEGINING OF THE STUDY (THIS IS PROBABLY WHAT HAPPENS HERE).

Our model was based on a number of assumptions and simplifications. These had to be realistic; otherwise, the model would not be useful and the parameter estimates would be biased. The assumption that temporary emigration was random (i.e., the probability of a bat being present at the colony depended on whether it was present one year before) was necessary so that we could use the recapture probabilities as estimates of the probability of presence of the different sex and age classes. Because nonreproducing females are often not present at the colony (this study) and reproduction is costly for female bats (Kurta et al. 1989; Kunz et al. 1995; Korine et al. 2004), it is possible that temporary absence from the colony depends on the reproductive success in the previous year and is thus a first-order Markov process. 
We assumed that the age at first reproduction was 2 years, even though some individuals of this species may reproduce for the first time only when they are 3 or more years old (Ransome 1995). We constructed a modified model in which we assumed that all individuals start to reproduce when they are three years old only, but this model was clearly worse than the best one $(\Delta \mathrm{DIC}=26.70)$.

We also assumed that immigration was nonexistent. Although we included all losses due to mortality and permanent emigration in our model, we only included gains through local recruitment. Immigration into the studied population was unlikely because the next colony of greater horseshoe bats was several hundreds of kilometers away in bat flight distance due to the high mountain ridges around the Rhône valley where our study colony was situated. Finally, our model was fairly simple in regards to the age structure of survival and fecundity rates.

These assumptions and simplifications were necessary to produce basic demographic information about this endangered bat species from sparse data. However, if the assumptions and simplifications have to be relaxed, or if finer patterns of the life history are detected, more data, in particular more years with recapture data, will be required. If more data were available, the integrated population model could easily be adapted to include nonrandom temporary emigration, probabilistic instead of deterministic age at first reproduction, and finer age structures in survival and fecundity. Clearly, a richer data set would also allow one to test for possible sex differences and temporal trends of vital rates with more power or to estimate temporal variation of the vital rates (hierarchical modeling with hyperparameters, e.g., Barry et al. 2003). Relaxing the assumption of no immigration can be achieved by specifying the model differentially. Because the immigrants are included in the counts, no additional data would be required to estimate immigration rate.

\section{Potential of integrated modeling}


Integrated population models are flexible tools that can be adapted to a variety of sampling situations. The strength of the integrated population model is that each model fragment borrows information from other model fragments, resulting in higher precision of parameter estimates and enabling estimation of parameters that could not be estimated otherwise (Besbeas et al. 2002). A further advantage of this framework is that it allows modeling of biologically plausible population processes and estimation of key biological parameters, while explicitly recognizing the uncertainties involved in the data collection (Besbeas et al. 2002; Buckland et al. 2004; Brooks et al. 2004; Besbeas et al. 2005). Once again, Buckland et al. 2004 do not deal with integrated population modeling. This ref. should be deleted. It is critical that the biological processes are well captured in the integrated model and that the count and the demographic data sets are independent. As demonstrated here, integrated population models allow one to estimate basic demographic parameters (fecundity in our example) that could otherwise not be estimated and its application resulted in much more accurate estimates of population growth and survival.

The level of confidence that one should give to each source of data is automatically handled with in the integrated population model. For example, the precision of survival rate is much poorer if estimated from count data alone compared to when capture-recapture data are used alone. The precision increases further, if both data types are used in conjunction to estimate survival, yet compared to the precision of survival from capture-recapture data alone the improvement is not so large. Thus, both types of data contain information about survival, but the quality is higher in the capture-recapture data than in the count data. Overall, the combination makes the sample size larger and hence increases precision and improves inference. This issue is explored in details in Brooks et al. (2004). OK, excellent!

In many monitoring systems the number of newborns and adults are recorded annually. Unless only reproducing females are included in the counts (which is rarely achieved in bats), these data would not be sufficient to estimate demographic parameters from an integrated 
population model. If, however, longitudinal data on marked individuals are available, estimation of demographic parameters is possible, as demonstrated in our example. It is not necessary to mark all young each year, and recapturing of adults does not necessarily have to be done on a yearly basis. In our example, the demographic parameters could have been estimated with one recapture event (i.e., without the data from year 2005), although parameter estimates would have been less precise. Not having to catch adults each year is appealing, especially if disturbance of the animals is a concern.

An important advantage of the integrated population models to monitoring is that a reliable estimate of fecundity can be obtained, which is particularly difficult in bats (Tuttle \& Stevenson 1982). The estimation requires, however, that the proportion of females present in a breeding population (e.g. a colony) be estimated. In most situations it will be impossible to capture all the adults in a population; therefore, the estimation of temporary emigration cannot be done as we did it. A possible solution is to perform at least two capture events in a year and to incorporate the robust design model (Kendall et al. 1997) into the integrated model. Another solution is to include a multistate model with an unobservable state (Schaub et al. 2004), but this requires that temporary emigration be nonrandom.

With an increasing number of species and populations at risk, an understanding of the ultimate causes of negative population trends in endangered organisms is needed. Carefully conducted demographic analyses have the potential to isolate the vital rates responsible for demographic variation. Targeted ecological, behavioral, or genetic investigations can then be performed to determine the proximate factors that effect (AFFECT?) the most crucial demographic parameters. This in turn will lead to accurate guidelines for the implementation of tailored conservation action plans. Given its potentially high resolution power, despite the scarcity of demographic data, integrated population models should become an essential tool of the modern conservation biologist. 


\section{Acknowledgements}

We thank the local authorities who provided free access to the bat roost, in particular the community and parish of Vex. The Conservation Service of the State of Valais and the Federal Environment Office funded the restoration of a bat-friendly church. O. G.'s research was supported by a Marie-Curie Intra-European Fellowship within the Sixth European Community Framework Programme. We thank F. Bontadina, M. Kéry, and B. Schmidt for their valuable comments on the manuscript. A. Lugon provided free access to his data. 


\section{Supplementary Material}

The WinBUGS code for the integrated population model, the demographic data of greater horseshoe bats from Vex (1991-2005), and the starting values to fit the most parsimonious model $\phi_{1}(),. \phi_{a}(),. f($.$) for reproducing the results are provided in conjunction with the online version$ of this paper at http:// xxx (I'll fill this in).

In your cover letter to Dr. Meffe, justify the inclusion of this supplementary material with the online version. Such material needs to be useful to researchers well into the future.

\section{Literature Cited}

Arlettaz R., A. Lugon, A. Sierro, and M. Desfayes. 1997. Les chauves-souris du Valais (Suisse): status, zoogéographie et écologie. Le Rhinolophe 12:1-42.

Baker G. B., L. F. Lumsden, E. B. Dettmann, N. K. Schedvin, M. Schulz, D. Natkins, and J. Jansen. 2001. The effect of forearm bands on insectivorous bats (Microchiroptera) in Australia. Wildlife Research 28:229-37.

Barry S. C., S. P. Brooks, E. A. Catchpole, and B. J. T. Morgan. 2003. The analysis of ringrecovery data using random effects. Biometrics 59:54-65.

Beissinger S. R. 2002. Population viability analysis: past, present, future. Pages 5-17 in S. R. Beissinger, editor. Population viability analysis. University of Chicago Press, Chicago. Besbeas P., S. N. Freeman, and B. J. T. Morgan. 2005. The potential of integrated population modelling. Australian and New Zealand Journal of Statistics 47:35-48. 
Besbeas P., S. N. Freeman, B. J. T. Morgan, and E. A. Catchpole. 2002. Integrating markrecapture-recovery and census data to estimate animal abundance and demographic parameters. Biometrics 58:540-7.

Besbeas P., J. D. Lebreton, and B. J. T. Morgan. 2003. The efficient integration of abundance and demographic data. Applied Statistics 52:95-102.

Boyd I. L., and R. E. Stebbings. 1989. Population changes of brown long-eared bats (Plecotus auritus) in bat boxes at Thetford forest. Journal of Applied Ecology 26:101-12.

Brooks S. P. 1998. Markov chain Monte Carlo method and its application. Journal of the Royal Statistical Society Series D - The Statistician 47:69-100.

Brooks S. P., and A. Gelman. 1998. Alternative methods for monitoring convergence of iterative simulations. Journal of Computational and Graphical Statistics 7:434-55.

Brooks S. P., R. King, and B. J. T. Morgan. 2004. A Bayesian approach to combining animal abundance and demographic data. Animal Biodiversity and Conservation 27.1:515-29.

Buckland S. T., K. B. Newman, L. Thomas, and N. B. Koesters. 2004. State-space models for the dynamics of wild animal populations. Ecological Modelling 171:157-75.

Burnham K. P. 1993. A theory for combined analysis of ring recovery and recapture data. Pages 199-213 in J. D. Lebreton, editor. Marked individuals in the study of bird population. Birkhäuser, Basel.

Burnham K.P., and D. R. Anderson. 1998. Model selection and inference. A practical information-theoretic approach. Springer, New York.

Caughley G. 1994. Directions in conservation biology. Journal of Animal Ecology 63:215-44.

Doak D. F., K. Gross, and W. F. Morris. 2005. Understanding and predicting the effects of sparse data on demographic analyses. Ecology 86:1154-63.

Duelli P. 1994. Listes rouges des espèces animals menaces de Suisse. Bundesamt für Umwelt, Wald und Landschaft, Bern, Switzerland. 
Gaisler J. 1989. The $r$ - $K$ selection model and life-history strategies in bats. Pages 117-24 in V.

Hanak, I. Horacek, and J. Gaisler, editors. European bat research 1987. Charles University Press, Praha, Czech Republic.

Gaisler J. 1966. A tentative ecological classification of colonies of the European bats. Lynx 6:359.

Gerell R., and K. Lundberg. 1990. Sexual differences in survival rates of adult pipistrelle bats (Pipistrellus pipistrellus) in South Sweden. Oecologia 83:401-4.

Hoyle S. D., A. R. Pople, and G. J. Toop. 2001. Mark-recapture may reveal more about ecology than about population trends: demography of a threatened ghost bat (Macroderma gigas) population. Austral Ecology 26:80-92.

Kendall W. L., J. D. Nichols, and J. E. Hines. 1997. Estimating temporary emigration using capture-recapture data with Pollock's robust design. Ecology 78:563-78.

Korine C., J. Speakman, and Z. Arad. 2004. Reproductive energetics of captive and free-ranging Egyptian fruit bats (Rousettus aegypticus). Ecology 85:220-30.

Kunz T. H., J. O. Whitaker Jr., and M. D. Wadanoli. 1995. Dietary energetics of the insectivorous Mexican free-tailed bat (Tadarida brasiliensis) during pregnancy and lactation. Oecologia 101:407-15.

Kurta A., G. P. Bell, K. A. Nagy, and T. H. Kunz. 1989. Energetics of pregnancy and lactation in free-ranging little brown bats (Myotis lucifugus). Physiolocical Zoology 62:804-18.

Lebreton J. D., K. P. Burnham, J. Clobert, and D. R. Anderson. 1992. Modeling survival and testing biological hypothesis using marked animals: a unified approach with case studies. Ecological Monographs 62:67-118.

Martin T. E., J. Clobert, and D. R. Anderson. 1995. Return rates in studies of life history evolution: are biases large? Journal of Applied Statistics 22:863-75. 
Maunder M. N. 2004. Population viability analysis based on combining Bayesian, integrated, and hierarchical analyses. Acta Oecologica 26:85-94.

Mickleburgh S. P., A. M. Hutson, and P. A. Racey. 2002. A review of the global conservation status of bats. Oryx 36:18-34.

Neuweiler G. 2000. The biology of bats. Oxford University Press, Oxford, United Kingdom. Norman A. P., G. Jones, and R. Arlettaz. 1999. Noctuid moths show neural and behavioural responses to sounds made by some bat-marking rings. Animal Behaviour 57:829-35.

Norris K. 2004. Managing threatened species: the ecological toolbox, evolutionary theory and declining-population paradigm. Journal of Applied Ecology 41:413-26.

O'Shea T. J., L. E. Ellison, and T. R. Stanley. 2004. Survival estimation in bats: historical overview, critical appraisal, and suggestions for new approaches. Pages 297-336 in W. L. Thompson, editor. Sampling rare and elusive species: concepts, designs and techniques for estimating population parameters. Island Press, Washington.

Pryde M. A., C. F. J. O'Donnell, and R. Barker. 2005. Factors influencing survival and long-term population viability of New Zealand long-tailed bats (Chalinolobus tuberculatus): implications for conservation. Biological Conservation 126:175-85.

R Development Core Team. 2004. R: a language and environment for statistical computing. R Foundation for Statistical Computing, Vienna, Austria.

Racey P. A. 1982. Ecology of bat reproduction. Pages 57-104 in T. H. Kunz, editor. Ecology of bats. Plenum Press, New York.

Ransome R. D. 1989. Population changes of greater horseshoe bats studied near Bristol over the past twenty-six years. Biological Journal of the Linnean Society 38:71-82.

Ransome R. D. 1995. Earlier breeding shortens life in female greater horseshoe bats. Philosophical Transitions of the Royal Society London B 350:153-61. 
Ransome R.D. 1990. The natural history of hibernating bats. Christopher Helm, London, United Kingdom.

Ransome R. D., and T. P. McOwat. 1994. Birth timing and population changes in greater horseshoe bat colonies (Rhinolophus ferrumequinum) are synchronized by climatic temperature. Zoological Journal of the Linnean Society 112:337-51.

Saether B. E., and O. Bakke. 2000. Avian life history variation and contribution of demographic traits to the population growth rate. Ecology 81:642-53.

Schaub M., O. Gimenez, B. R. Schmidt, and R. Pradel. 2004. Estimating survival and temporary emigration in the multistate capture-recapture framework. Ecology 85:2107-13.

Sendor T., and M. Simon. 2003. Population dynamics of the pipistrelle bat: effects of sex, age and winter weather on seasonal survival. Journal of Animal Ecology 72:308-20.

Spiegelhalter D., A. Thomas, and N. G. Best. 2004. WinBUGS user Manual. Version 1.4. MCR Biostatistics Unit, Cambridge, United Kingdom.

Spiegelhalter D. J., N. G. Best, B. P. Carlin, and A. van der Linde. 2002. Bayesian measure of model complexity and fit. Journal of the Royal Statistical Society Series B 64:583-639. Stebbings R.E. 1988. Conservation of European bats. Christopher Helm, London, United Kingdom.

Sturtz S., U. Ligges, and A. Gelman. 2005. R2WinBUGS: a package for running WinBUGS from R. Journal for Statistical Software 12:1-16.

Tuttle M. D., and D. Stevenson. 1982. Growth and survival of bats. Pages 105-50 in T. H. Kunz, editor. Ecology of bats. Plenum Press, New York.

van Groenendael J., H. de Kroon, and H. Caswell. 1988. Projection matrices in population biology. Trends in Evolution \& Ecology 3:264-9.

Warren R. D., and M. S. Witter. 2002. Monitoring trends in bat populations through roost surveys: methods and data from Rhinolophus hipposideros. Biological Conservation 105:255-61. 
Table 1. Modeling results of different integrated population models of greater horseshoe bats from the Vex colony (1991-2005).*

\begin{tabular}{|c|c|c|c|}
\hline Model & Deviance & $\mathrm{pD}$ & $\Delta \mathrm{DIC}$ \\
\hline$\phi_{1}(),. \phi_{a}(),. f()$. & 261.69 & 28.07 & 0.00 \\
\hline$\phi_{1}(s), \phi_{a}(),. f()$. & 262.21 & 28.38 & 0.83 \\
\hline$\phi_{1}(),. \phi_{a}(s), f()$. & 262.16 & 28.81 & 1.22 \\
\hline$\phi_{1}(),. \phi_{a}(),. f(T)$ & 262.43 & 28.59 & 1.27 \\
\hline$\phi_{1}(),. \phi_{a}(T), f()$. & 262.54 & 28.59 & 1.37 \\
\hline$\phi_{1}(),. \phi_{a}(T), f(T)$ & 263.33 & 28.96 & 2.53 \\
\hline$\phi_{1}(s), \phi_{a}(s), f()$. & 263.06 & 29.71 & 3.01 \\
\hline$\phi_{1}(s), \phi_{a}(),. f(T)$ & 263.26 & 29.65 & 3.15 \\
\hline$\phi_{1}(T), \phi_{a}(),. f()$. & 262.29 & 30.66 & 3.19 \\
\hline$\phi_{1}(),. \phi_{a}(s), f(T)$ & 263.18 & 29.91 & 3.33 \\
\hline$\phi_{1}(s), \phi_{a}(s), f(T)$ & 264.01 & 30.82 & 5.07 \\
\hline$\phi_{1}(T), \phi_{a}(),. f(T)$ & 263.25 & 31.89 & 5.38 \\
\hline$\phi_{1}(T), \phi_{a}(T), f(T)$ & 264.12 & 32.82 & 7.18 \\
\hline$\phi_{1}(T), \phi_{a}(T), f()$. & 263.22 & 34.58 & 8.04 \\
\hline$\phi_{1}(t), \phi_{a}(t), f(t)$ & 215.35 & 728.38 & 653.97 \\
\hline
\end{tabular}

* The model parameters are the age-specific survival rates $\left(\phi_{1}:\right.$ juveniles, $\phi_{a}$ : adults) and fecundity rate (f). The other parameters in the models, the population sizes and the recapture 
rates, were in all models time-, sex-and age-specific. The notation (T) refers to a linear time trend of the specific parameter, $(t)$ indicates year-specific rates, $(s)$ refers to sex specific rates, and (.) denotes constancy. The model deviance, model complexity ( $p D)$, and difference of the deviance information criterion between the best and the current model ( $\triangle D I C)$ are provided. Models are ranked according to their support of the data; the best supported model is at the top. 
Table 2. Estimated demographic parameters of greater horseshoe bats from the Vex colony $(1991-2005) *{ }^{*}$

\begin{tabular}{lcccc}
\hline & Mean & SD & Lower & Upper \\
& & & & \\
\hline Juvenile survival $\left(\phi_{1}\right)$ & 0.489 & 0.064 & 0.371 & 0.619 \\
Adult survival $\left(\phi_{a}\right)$ & 0.906 & 0.020 & 0.865 & 0.943 \\
Fecundity $(f)$ & 0.743 & 0.115 & 0.562 & 1.008 \\
Presence of subadult females $\left(\tau_{1}^{f}\right)$ & 0.821 & 0.105 & 0.575 & 0.974 \\
Presence of adult females $\left(\tau_{a}^{f}\right)$ & 0.919 & 0.042 & 0.821 & 0.981 \\
Presence of subadult males $\left(\tau_{1}^{m}\right)$ & 0.675 & 0.140 & 0.393 & 0.927 \\
Presence of adult males $\left(\tau_{a}^{m}\right)$ & 0.282 & 0.093 & 0.131 & 0.494 \\
Census variance $\left(\sigma_{y}^{2}\right)$ & & & & \\
\end{tabular}

* Given are the posterior mean, standard deviation (SD) and limits of the $95 \%$ credible intervals (lower, upper) of demographic parameters estimated with the most parsimonious model $\left(\phi_{1}(),. \phi_{a}(),. f().\right)$ 
Figure captions

Figure 1. Directed acyclic graph (DAG) of the integrated population model for greater horseshoe bats in the Vex colony. Estimated parameters are represented by circles, and the data are represented by rectangles. Arrows represent dependences between nodes. To simplify the graph, the different sex and age classes are represented by one node $N$. Node notations: $m$, capturerecapture data; $y$ : count data, $J$ : number of newborns; $f$ : fecundity rate; $\phi_{x}^{k}$ : local survival rate of bats in age class $x$ with sex $k$ (where 1 , first year; ad, adult; $\mathrm{m}$, male; $\mathrm{f}$, female); $p_{x}^{k}$ : recapture rate of bats in age class $x$ with sex $k$ (where 1, first year; ad, adult; m, male; f, female); $N$ : population size; $\sigma_{y}^{2}$ : variance of the count.

Figure 2. Annual population surveys (counts) of greater horseshoe bats at emergence of the roost in Vex (no survey was carried out in 1991 and 2001) and the annual number of newborn greater horseshoe bats in Vex.

Figure 3. Estimated population size of greater horseshoe bat colony in Vex stratified according to sexes and age classes based on the most parsimonious model $\left(\phi_{1}(),. \phi_{a}(),. f().\right)$. Bars are the posterior mean, and the vertical lines represent the limits of the $95 \%$ credible intervals.

Figure 4. Annual population growth rates of greater horseshoe bats in Vex estimated based on the raw counts (population surveys) and on the integrated population model (IP model). The closed dots from the IP model are posterior means based on the most parsimonious model $\left(\phi_{1}(),. \phi_{a}(),. f().\right)$; the vertical lines show the limits of the $95 \%$ credible interval. 


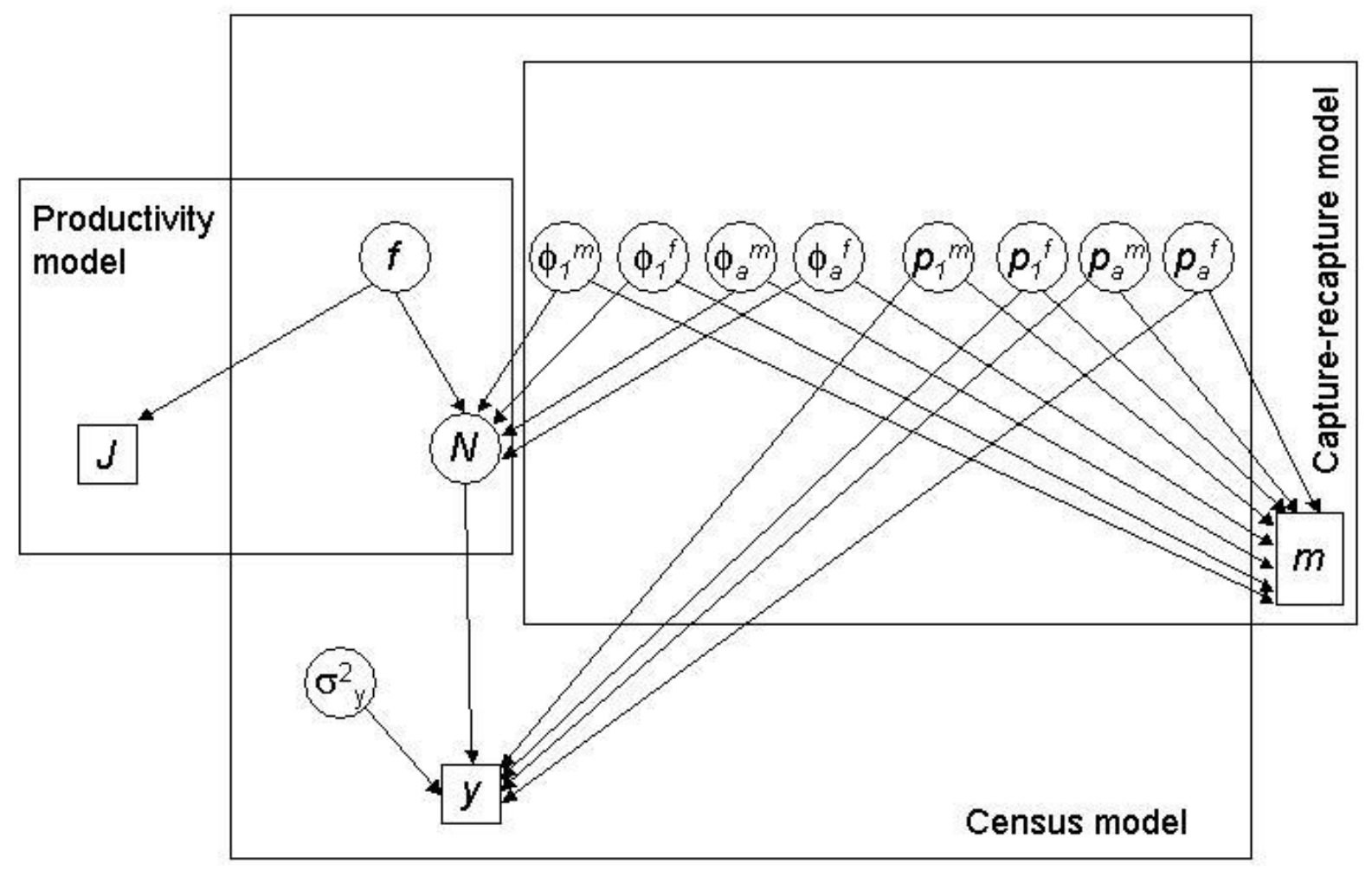




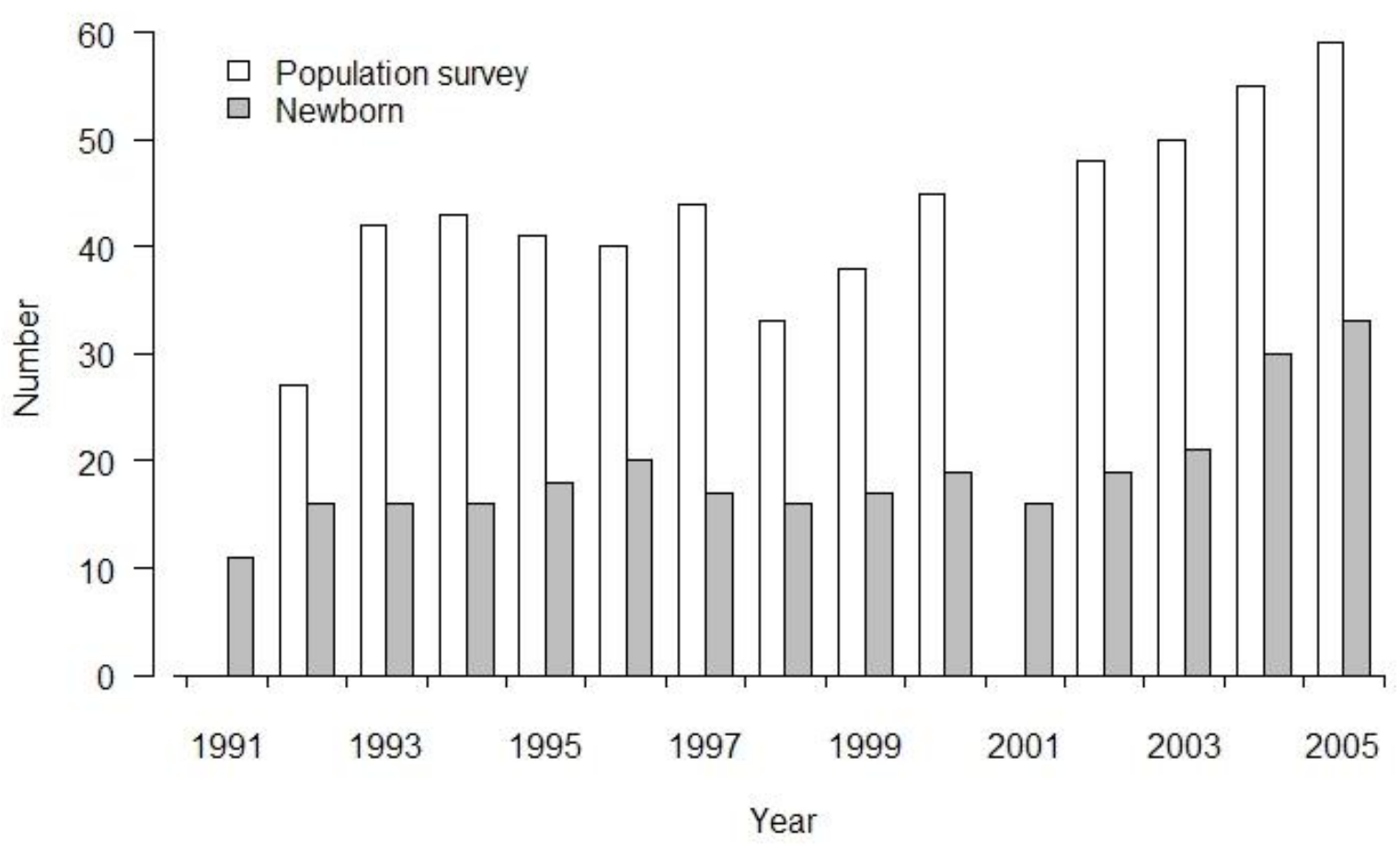


Subadult females

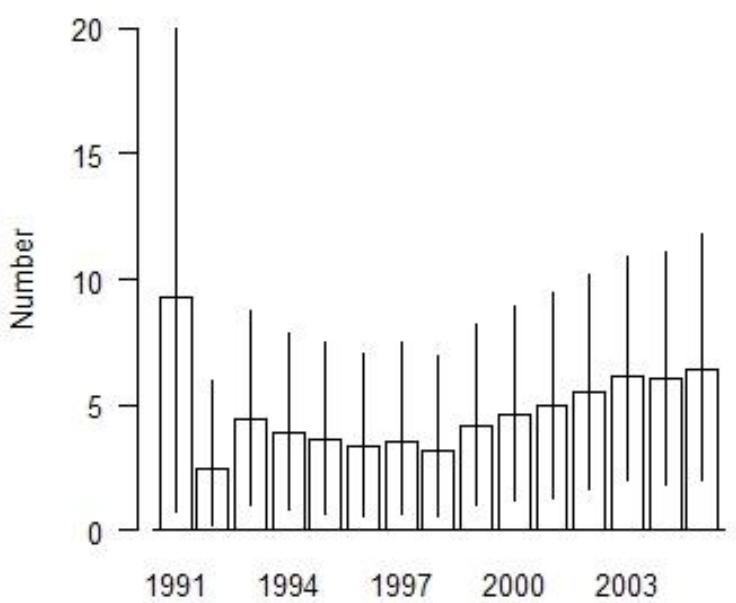

Adult females

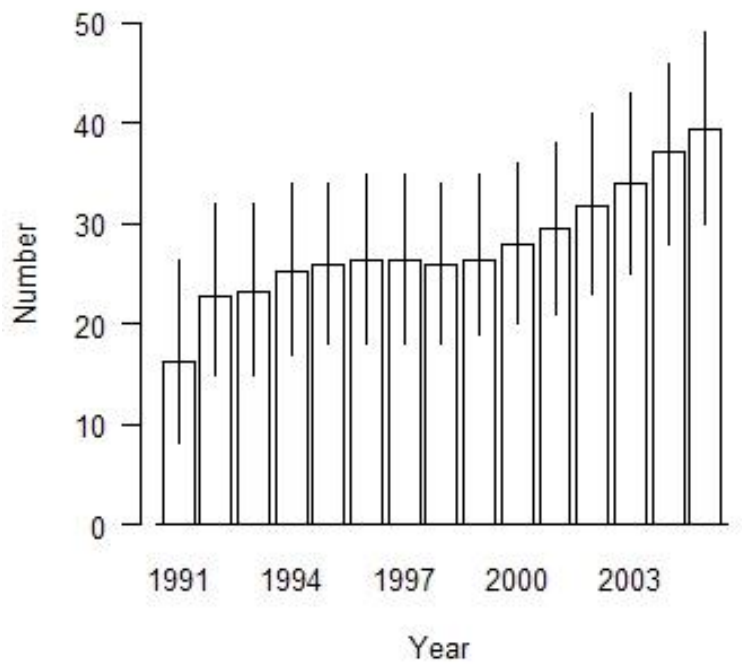

Subadult males

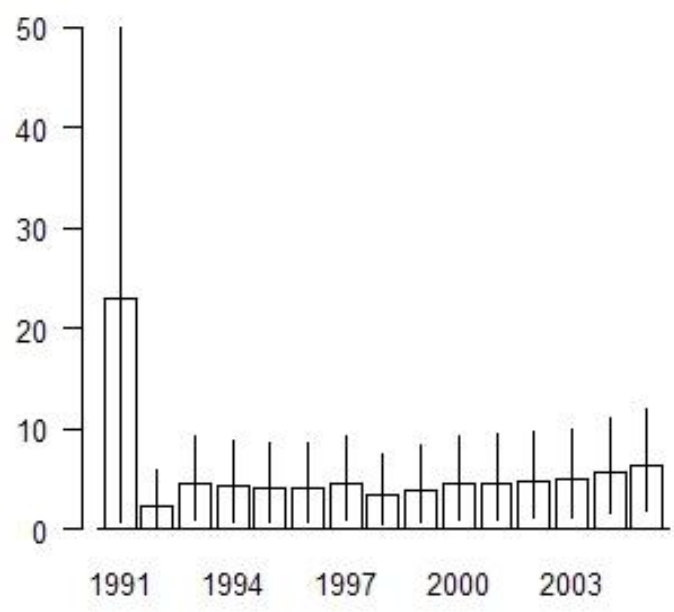

Adult males

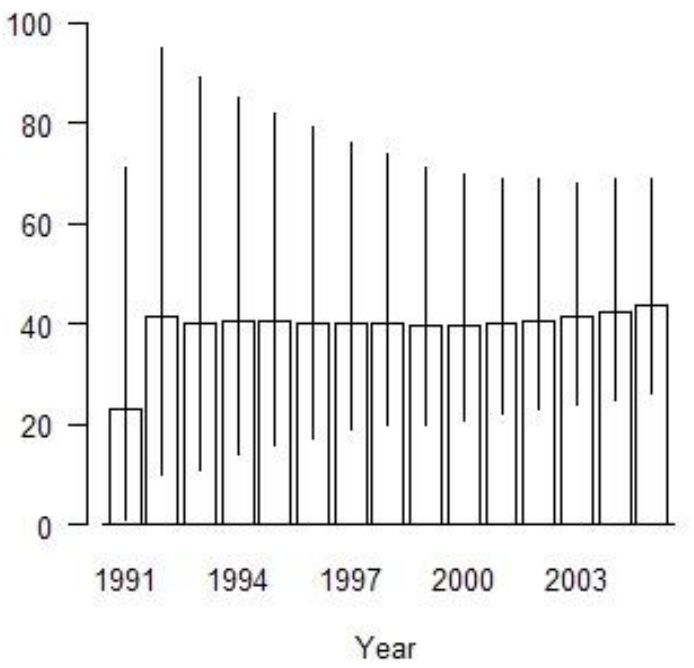




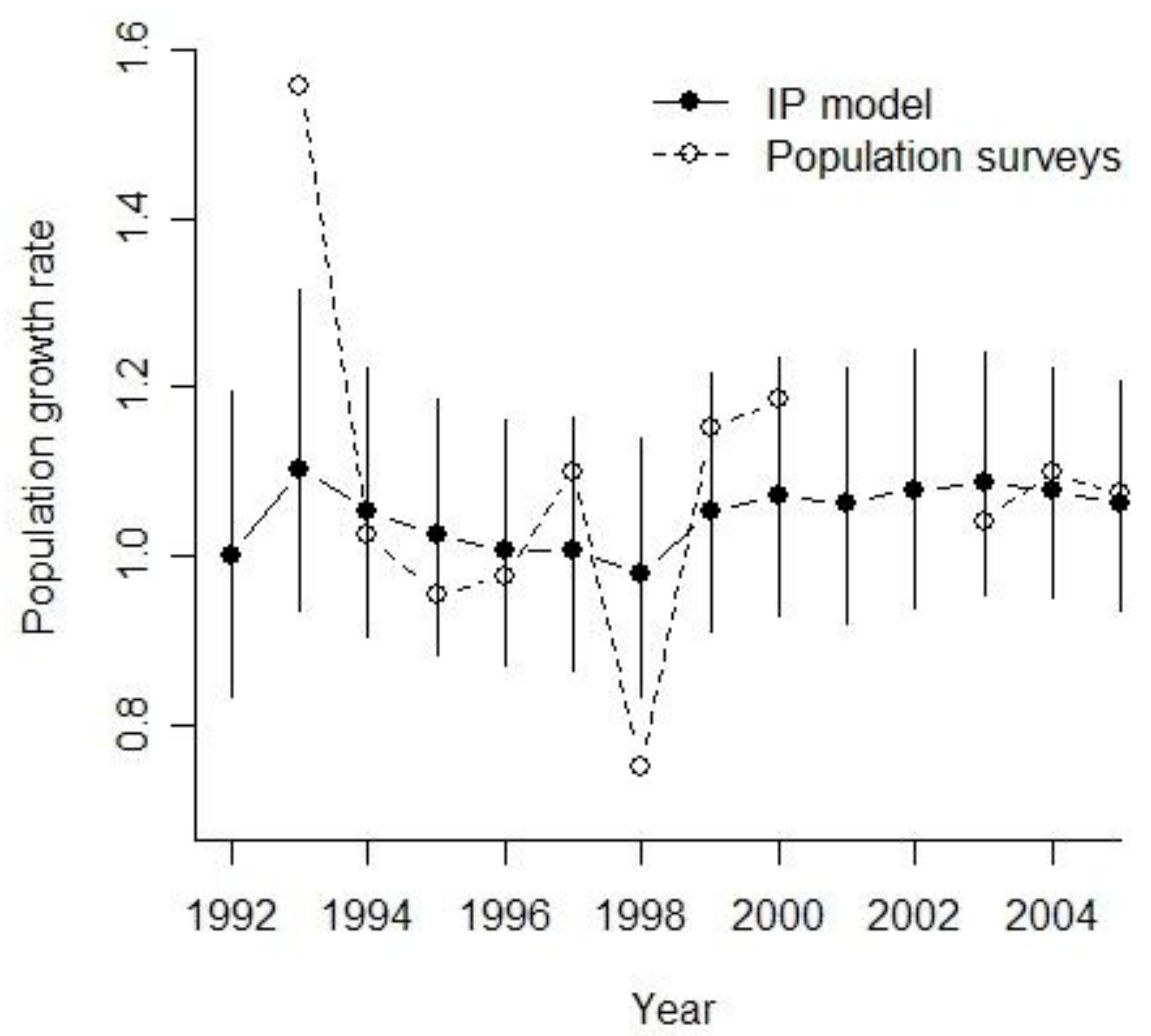

